\title{
Structure illumination microscopy imaging of lipid vesicles in live bacteria with naphthalimide-appended organometallic complexes
}

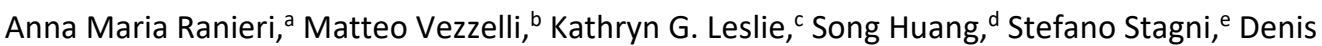 \\ Jacquemin, ${ }^{f}$ Haibo Jiang, ${ }^{d}$ Alysia Hubbard, ${ }^{d}$ Luca Rigamonti, ${ }^{b}$ Elizabeth L. J. Watkin, ${ }^{\mathrm{g}}$ Mark I. \\ Ogden, ${ }^{* a}$ Elizabeth J. New, ${ }^{* c}$ and Massimiliano Massi*a
}

There is a lack of molecular probes for imaging bacteria, in comparison to the array of such tools available for the imaging of mammalian cells. Here, organometallic molecular probes have been developed and assessed for bacterial imaging, designed to have the potential to support multiple imaging modalities. The chemical structure of the probes is designed around a metalnaphthalimide structure. The 4-amino-1,8-naphthalimide moiety, covalently appended through a pyridine ancillary ligand, acts as a luminescent probe for super-resolution microscopy. On the other hand, the metal centre, rhenium(I) or platinum(II) in the current study, enables techniques such as nanoSIMS. While the rhenium(I) complex was not sufficiently stable to be used as probe, the platinum(II) analogue showed good chemical and biological stability. Structured illumination microscopy (SIM) imaging on live Bacillus cereus confirmed the suitability of the probe for superresolution microscopy. NanoSIMS analysis was used to monitor the uptake of the platinum(II) complex within the bacteria and demonstrate the potential of this chemical architecture to enable multimodal imaging. The successful combination of these two moieties introduces a platform that could lead to a versatile range of multi-functional probes for bacteria.

Sub-cellular imaging of bacteria is challenging but important. Antibiotic resistance and infections prevention are pressing

\footnotetext{
a. School of Molecular and Life Sciences, Curtin University, Bentley 6102 WA,

Australia.m.massi@curtin.edu.au,m.ogden@curtin.edu.au.

b. Dipartimento di Scienze Chimiche e Geologiche, Università degli Studi di Modena e Reggio Emilia, via G. Campi 103, 41125 Modena, Italy

School of Chemistry, The University of Sydney, 2006 NSW, Australia. elizabeth.new@sydney.edu.au

d. Centre for Microscopy, Characterisation and Analysis, University of Western Australia, 6009 Perth WA, Australia.

e. Dipartimento di Chimica Industriale "Toso Montanari", Università degli Studi di Bologna, viale del Risorgimento 4, 40136 Bologna, Italy.

f. Laboratoire CEISAM, UMR CNRS 6230, Universit8 de Nantes, 2 Rue de la HoussiniHre, BP 92208, 44322 Nantes Cedex 3, France.

g. Curtin Medical School, Curtin University, Kent Street, Bentley 6102 WA, Australia
}

Electronic Supplementary Information (ESI) available: synthetic detailes, NMR, photophysical measurements, theoretical calculations, bacterial growth and imaging details. health concerns, ${ }^{1}$ and bacteria are also used for the natural synthesis of biopolymers and are potential alternative sources for oil and fuel production. Where it has been achieved, high resolution imaging of bacterial cells has contributed to great advances in prokaryotic cell biology, enabling study of the subcellular structures that play a key role in the growth and pathogenesis of these unicellular organisms. ${ }^{2}$ Given the small size of bacterial cells, super-resolution microscopy is pivotal to successful visualisation of microbial specimens with sub-cellular resolution. ${ }^{3}$ Most of the fluorescence imaging on bacterial cells has to date been achieved using genetically encoded tags or commercially available dyes. Given the lack of small-molecule probes that can readily penetrate the cell envelope of live bacteria and target cellular sub-structures, considerable recent research effort has focussed on the development of new fluorophores designed to efficiently stain live bacteria. ${ }^{2,4}$ While most of these fluorophores are small organic molecules, ${ }^{2 b, 3,5}$ we have recently reported a sequence of phosphorescent iridium complexes that selectively stain lipid vacuoles within bacteria. ${ }^{4 \mathrm{~d}}$

Here, our aim was to develop probes bearing the 4-amino1,8-naphthalimide fluorophore that enable structured illumination microscopy (SIM) imaging, while also incorporating heavy metal ions that could support additional imaging methods, ${ }^{6}$ or other functionality such as antibacterial properties. In this proof of concept work, the metal ion was tracked using nano-scale secondary ion mass spectrometry (nanoSIMS). ${ }^{7}$ NanoSIMS is particularly well-suited to analysis of isotopes and trace elements ${ }^{8}$ and has been combined with confocal microscopy to monitor the cellular uptake and subcellular accumulation of platinum-based drugs in human cells and tissues. ${ }^{9}$ NanoSIMS protocols previously applied to bacteria have generally relied on the more traditional approach based on the stable isotope labelling of target biological substrates. ${ }^{10}$ We report the synthesis and characterisation of rhenium(I) and platinum(II) complexes bound to naphthalimide-appended ligands (Scheme 1). Similar complexes of rhenium(I) were previously investigated by Pope et al. in human osteoarthritic, protistan fish parasite Spironucleus vortens and fission yeast 
Schizosaccharomyces pombe cells using confocal fluorescence miscroscopy, ${ }^{11}$ but never assessed for live bacterial imaging. In the present study, we determined that the stability of the rhenium(I) complex was not sufficient for synthesis and application as a biological probe, bacterial imaging was studied using exclusively the platinum(II) complex. Pt-PyNapht was tested to image the live bacterial species $B$. cereus to validate the capability of this system to enable structure illumination microscopy, with the presence of the metal confirmed by nanoscale secondary ion mass spectrometry.
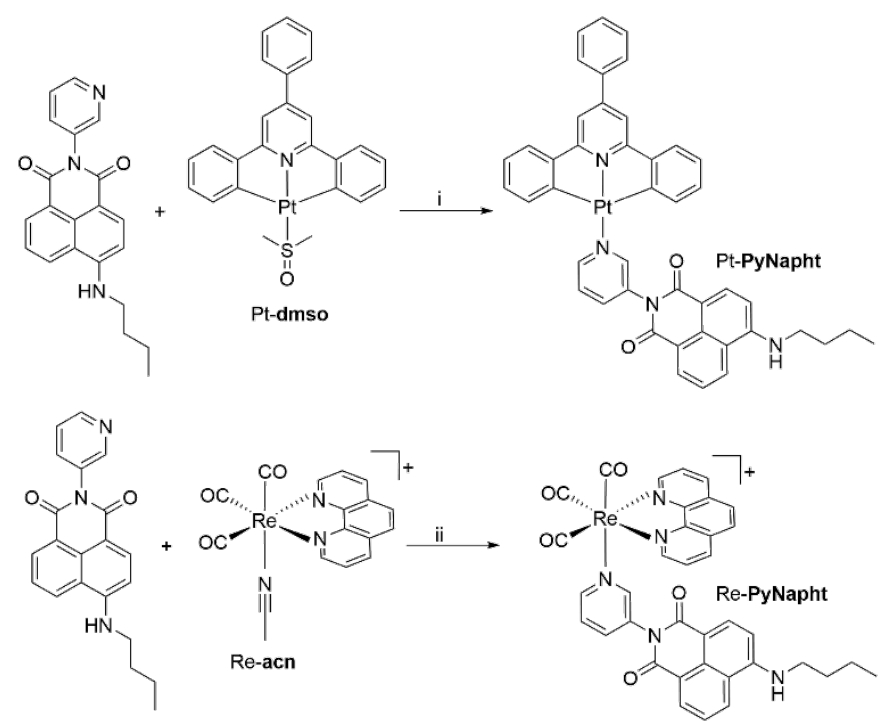

Scheme 1. Synthesis of the targeted complex Pt-PyNapht and Re-PyNapht. Reagents and conditions: i) Acetone/MeOH (9:1 v/v), reflux, overnight; ii) $\mathrm{CH}_{3} \mathrm{CN}$, reflux, overnight.

The synthetic routes for the formation of the complexes PtPyNapht and Re-PyNapht are described in Scheme 1, and further synthetic details are summarised in the ESI. The dicyclometalated platinum(II) precursor Pt-dmso was synthesised from the corresponding dichloro-bridged dimer, following a previously reported method, ${ }^{12}$ whereas the cationic tricarbonyl diimine rhenium(I) precursor was prepared in situ by reaction of $\left[\operatorname{Re}(\mathrm{CO})_{3}\right.$ (phen) $\left.\mathrm{Br}\right]$ (phen $=1,10$-phenanthroline) with $\mathrm{AgBF}_{4}$ in acetonitrile at reflux. The $\mathrm{N}$-3-pyridyl-4butylamino-1,8-naphthalimide ligand (PyNapht) was prepared by reacting 4-nitro-naphthalic anhydride with 3-aminopyridine and butylamine (see ESI). ${ }^{13}$ PyNapht was then made to react with the corresponding precursors to afford the target compounds via a ligand exchange reaction, with moderate yields for the platinum(II) complex (35\%) and very low yield for the rhenium(I) complex, usually after successive flash chromatography to obtain a pure sample (7\%). The complexes Pt-PyNapht and Re-PyNapht were characterised by NMR spectroscopy and elemental analysis, confirming the expected structures.

The photophysical properties of the complexes are summarised in Table 1. Due to stability issues, the data for RePyNapht are only reported in $\mathrm{CH}_{2} \mathrm{Cl}_{2}$. The absorption and emission spectra of Pt-PyNapht in $\mathrm{CH}_{2} \mathrm{Cl}_{2}$ are depicted in Figure 1 , whereas the corresponding spectra for the rhenium(I) complex are reported in the ESI. The photophysical properties of the rhenium(I) complex are analogous to previously published similar complexes, indicating that the presence or absence of a methylene spacer between the pyridine ring and naphthalimide moiety in the ancillary ligand has very little effect. ${ }^{11 a}$ The Pt-PyNapht complex exhibits a strong absorption band centred at $282 \mathrm{~nm}\left(\varepsilon=15.26 \times 10^{4} \mathrm{M}^{-1} \mathrm{~cm}^{-1}\right)$ followed by a band at $363 \mathrm{~nm}$ of lower intensity $\left(\varepsilon=3.55 \times 10^{4} \mathrm{M}^{-1} \mathrm{~cm}^{-1}\right)$ that are assigned to $\pi-\pi^{*}$ transitions of the triphenylpyridine ligand. ${ }^{14} \mathrm{~A}$ further red-shifted band is observed at $433 \mathrm{~nm}(\varepsilon=$ $5.04 \times 10^{4} \mathrm{M}^{-1} \mathrm{~cm}^{-1}$ ), and is assigned to the lowest dipoleallowed transition in the 4-amino-1,8-naphthalimide, a transition presenting a partial charge-transfer (CT) character. ${ }^{11 a, 15}$ These assignments are confirmed by $a b$ initio calculations (see the ESI). A very similar absorption band was observed by recording the absorption spectra of the uncoordinated PyNapht in the same solvent (see the ESI), showing that the contribution of this lower energy band is mainly ascribed to the naphthalimide moiety. The absorption spectra of Pt-PyNapht in DMSO and $\mathrm{H}_{2} \mathrm{O}$ followed the same trend (see the ESI).
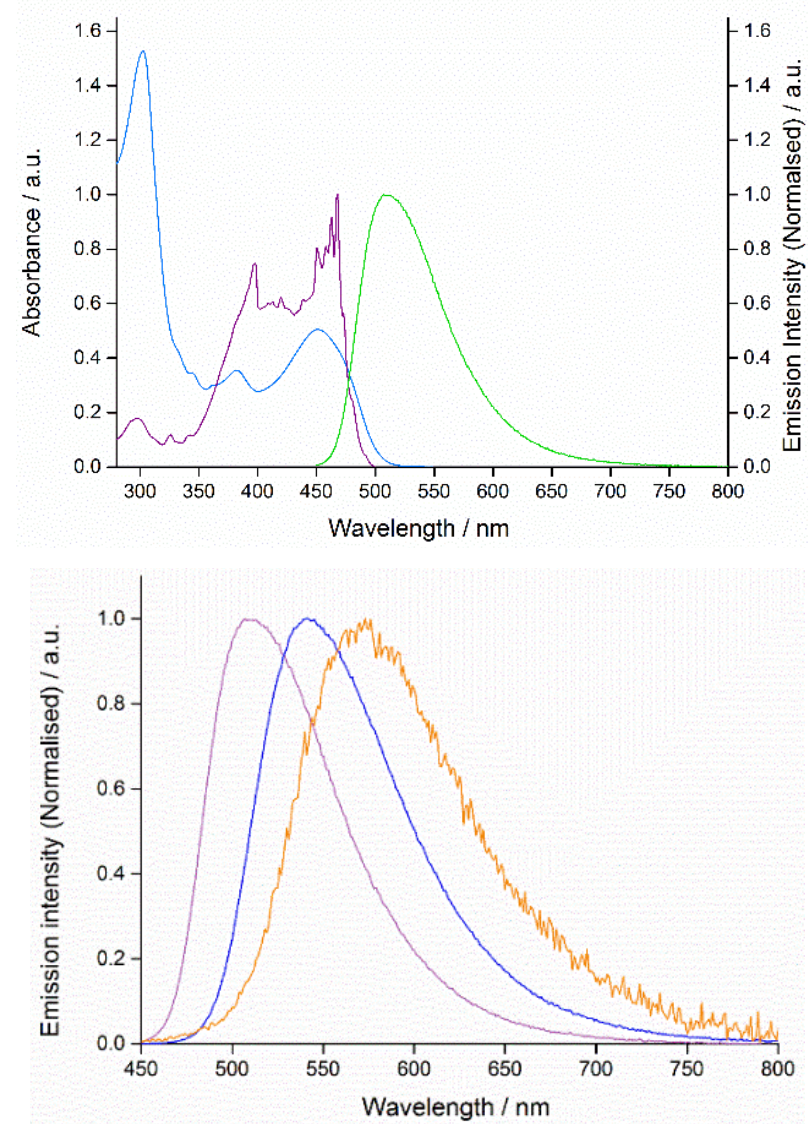

Figure 1. Top: Absorption (blue line), normalised excitation (purple line, $\lambda_{\mathrm{em}}=515 \mathrm{~nm}$ ) and normalised emission (green line, $\lambda_{\text {exc }}=430 \mathrm{~nm}$ ) spectra of Pt-PyNapht from a $10^{-5} \mathrm{M}$ $\mathrm{CH}_{2} \mathrm{Cl}_{2}$ solution. Bottom: Normalised emission spectra $\left(\lambda_{\text {exc }}=430 \mathrm{~nm}\right)$ of Pt-PyNapht in $\mathrm{CH}_{2} \mathrm{Cl}_{2}$ (violet line), DMSO (blue line), $\mathrm{H}_{2} \mathrm{O}$ (orange line). 
Table 1. Photophysical data of Pt-PyNapht and Re-PyNapht from dilute $\left(10^{-5} \mathrm{M}\right)$ solutions.

\begin{tabular}{|c|c|c|c|c|}
\hline Complex & $\begin{array}{c}\lambda_{\mathrm{abs}} / \mathrm{nm} \\
\left(10^{4} \varepsilon\left[\mathrm{M}^{-1} \mathrm{~cm}^{-1}\right]\right)\end{array}$ & $\begin{array}{c}\lambda_{\mathrm{em}} \\
{[\mathrm{nm}]}\end{array}$ & $\begin{array}{c}\tau_{\mathrm{aer}} \\
{[\mathrm{ns}]}\end{array}$ & $\begin{array}{c}\Phi_{\text {aer }}^{(a)} \\
\%\end{array}$ \\
\hline $\begin{array}{l}\text { Pt-PyNapht } \\
\qquad\left(\mathrm{CH}_{2} \mathrm{Cl}_{2}\right)\end{array}$ & $\begin{array}{c}282(15.26), 363 \\
(3.55), \\
433(5.04)\end{array}$ & 515 & 11 & 28 \\
\hline $\begin{array}{l}\text { Pt-PyNapht } \\
\text { DMSO }\end{array}$ & $\begin{array}{c}287(11.02), 348 \\
(2.60), \\
448(3.61)\end{array}$ & 542 & 10 & 5 \\
\hline $\begin{array}{l}\text { Pt-PyNapht } \\
\qquad\left(\mathrm{H}_{2} \mathrm{O}\right)^{(\mathrm{b})}\end{array}$ & $\begin{array}{c}284(7.59), 365 \\
(2.31) \\
461(2.10)\end{array}$ & 572 & 3 & 2 \\
\hline $\begin{array}{c}\text { Re-PyNapht } \\
\quad\left(\mathrm{CH}_{2} \mathrm{Cl}_{2}\right)\end{array}$ & $\begin{array}{c}276(3.17), 435 \\
(1.05)\end{array}$ & 517 & $\begin{array}{c}5(57 \%) \\
10(43 \%)\end{array}$ & 25 \\
\hline
\end{tabular}

(a) Measured vs $\left[\mathrm{Ru}(\mathrm{bpy})_{3}\right]^{2+}$ in $\mathrm{H}_{2} \mathrm{O}\left(\phi_{\mathrm{r}}=0.028\right)$. (b) $0.1 \%$ DMSO was added to favour the solubilisation of the complex.

With increasing solvent polarity, a red-shift was observed for the lowest energy band, shifting from around $433 \mathrm{~nm}$ to 461 $\mathrm{nm}$ moving from $\mathrm{CH}_{2} \mathrm{Cl}_{2}$ to $\mathrm{H}_{2} \mathrm{O}$. This behaviour is ascribed to a strong solvatochromism that is typical of excited states of CT nature. ${ }^{13,15 b}$

Excitation of a $\mathrm{CH}_{2} \mathrm{Cl}_{2}$ solution of Pt-PyNapht at $430 \mathrm{~nm}$ resulted in a broad emission band centred at $515 \mathrm{~nm}$, again typical of the CT emissive state of 1,8-naphthalimide fluorophores. The excitation spectrum matched the CT absorption band, suggesting that no electronic interaction existed between the platinum centre and the naphthalimide ligand.11a,15a Moreover, the fluorescence lifetime recorded at the emission maximum exhibited a value of $11 \mathrm{~ns}$, closely matching the value observed for the uncoordinated PyNapht (see the ESI). These observations suggest that the emission observed for Pt-PyNapht is dominated by the fluorescence of the 1,8-naphthalimide, in agreement with previously reported $\operatorname{Re}(\mathrm{I})$ and $\mathrm{Pt}$ (II) complexes bearing related 1,8-naphthalimide ligands. ${ }^{11 a, 15 a}$ As observed in the absorption spectra, a positive solvatochromism occurred with increasing solvent polarity, with a red-shift of $57 \mathrm{~nm}$ from $\mathrm{CH}_{2} \mathrm{Cl}_{2}$ to $\mathrm{H}_{2} \mathrm{O}$ (Figure 1). The fact that the properties of the naphthalimide are unchanged upon coordination to the rhenium(I) or platinum(II) centre is particularly advantageous, as it allows the photophysical properties of the luminescent moiety to be selected and tuned independently from the metal centre, which is acting as a "silent" carrier.

To further investigate the structural and photophysical properties of PyNapht and Pt-PyNapht, we performed timedependent density functional theory (TD-DFT) calculations, using a protocol described in the ESI. For PyNapht, the lowest state can be ascribed to a $\pi-\pi^{*}$ transition, involving a CT of 0.60 e over $2.10 \AA$. The computed $0-0$ energy is $2.77 \mathrm{eV}, 0.19 \mathrm{eV}$ off the experimental value $(2.58 \mathrm{eV})$, a mismatch typical for TDDFT. ${ }^{16}$ In Pt-PyNapht, the dihedral angle between the pyridyl ring and the 1,8-naphthalimide (PT complex) plane is $62^{\circ}\left(54^{\circ}\right)$. This leads to a near orthogonality of the two chromophores (angle of $86.2^{\circ}$, see the ESI), preventing direct electronic interactions. The TD-DFT calculations reveal that the lowest singlet excited state in Pt-PyNapht is located on the 1,8naphthalimide moiety (no lower CT-like state is found) and presents a 0-0 energy of $2.78 \mathrm{eV}$, almost identical to that determined in PyNapht. Overall, theoretical calculations therefore confirm that there is almost no interaction between the two moieties in Pt-PyNapht and that the lowest excited state is always located on the naphthalimide.

The use of Pt-PyNapht as a molecular probe for fluorescence microscopy in live bacteria was investigated by structured illumination microscopy (SIM). The complex was incubated with live $B$. cereus directly in the growth medium (Nutrient Broth, 0.1\% DMSO), and the fluorescence of PtPyNapht was easily detected upon excitation with a $488 \mathrm{~nm}$ laser after only $15 \mathrm{~min}$ incubation (Figure 2). To gain further spatial information about the interaction of the complex with $B$. cereus, bacteria were co-stained with MitoTracker Red. This commercial dye specifically stains the mitochondria in mammalian eukaryotic cells and while its use to label bacteria is quite limited, ${ }^{17}$ we show here that MitoTracker could stain live $B$. cereus in the growth medium and enabled simultaneous staining with Pt-PyNapht. Notably, the super-resolution images showed that the complex exhibited sub-cellular localisation in well-defined inclusions, whose shape resembled lipid bodies. As reported previously, ${ }^{4 d}$ we confirmed the lipid nature of these organelles by staining with the lipophilic dye BODIPY $493 / 503$ (see the ESI). The uncoordinated ligand PyNapht exhibited the same sub-cellular localisation as Pt-PyNapht (see the ESI), in line with related 4-amino1,8-naphthalimides that showed preferential affinity for lipid droplets in eukaryotic cells. ${ }^{13}$ The staining experiments suggest that while the platinum(II) dicyclometalated fragment does not affect the sub-cellular localisation of the 4-amino-1,8-naphthalimide unit, adding the metal centre to the dye provides the additional opportunity to detect the probe with electron or isotope-based techniques not readily accessible for organic dyes. In addition, very few probes have been reported to date for use in super-resolution microscopy. Hence, the detectability of Pt-PyNapht, as well as the uncoordinated PyNapht, by structured illumination microscopy opens the potential development of new classes of dyes for super-resolution microscopy.
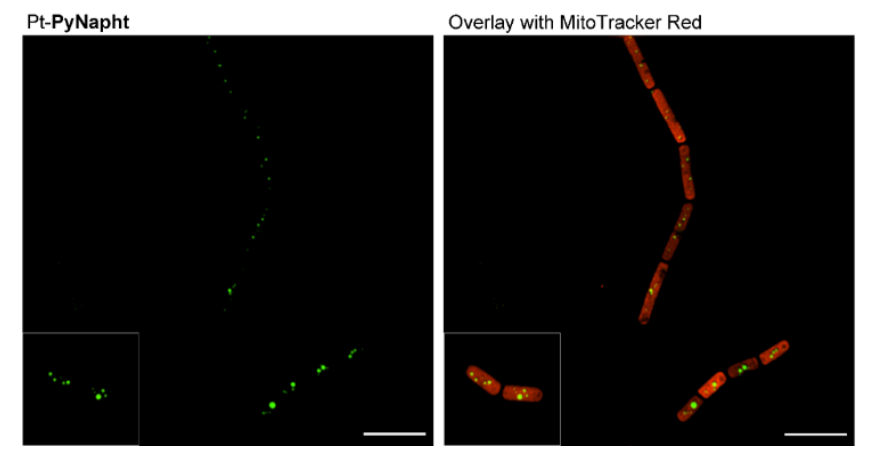

Figure 2. SIM images of live B. cereus incubated with Pt-PyNapht (left) and co-stained with MitoTracker Red (right). Pt-PyNapht was excited at $488 \mathrm{~nm}$ and the emission collected in the 525/50 $\mathrm{nm}$ region. MitoTracker Red was excited at $561 \mathrm{~nm}$ and the emission collected in the 605/50 region. Scale bars: $5 \mu \mathrm{m}$. 
The presence of platinum introduced using Pt-PyNapht was assessed using nanoSIMS to confirm the uptake of the platinumbased dye in the bacteria. ${ }^{12} \mathrm{C}^{14} \mathrm{~N}^{-}$secondary ions were used to show the morphology of single bacteria, and ${ }^{195} \mathrm{Pt}^{-}$secondary ion was used to indicate the distributions of Pt-PyNapht (Figure 3). The resulting nanosIMS images confirmed the internalisation of Pt-PyNapht within B. cereus. The distribution of Pt-PyNapht in these cells is not homogenous, and there are some subcellular regions that yield higher ${ }^{195} \mathrm{Pt}^{-}$signals. It should be noted that nanoSIMS images were generated from the top $<50 \mathrm{~nm}$ of the section whereas signals of Pt-PyNapht in structural illumination microscope images were obtained from entire bacteria. Nevertheless, the images are consistent enough with the SIM images to suggest that the platinum has been retained in the probe molecule.
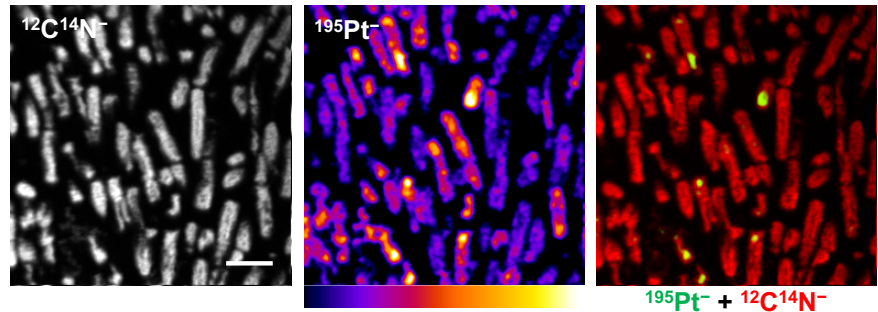

Figure 3. NanoSIMS images of $B$. cereus incubated with Pt-PyNapht for 15 minutes. Cells were fixed, dehydrated and embedded in resin. Semi-thin sections were cut for NanoSIMS imaging. ${ }^{195} \mathrm{Pt}^{-}$image was processed with a mean filter with a radius of 3 pixels. The threshold of ${ }^{195} \mathrm{Pt}^{-}$in the composite image is set to show the features with highest ${ }^{195} \mathrm{Pt}^{-}$signals in B. cereus. Scale bars: $3 \mu \mathrm{m}$.

This work demonstrates that the small molecule probe PtPyNapht efficiently stains $B$. cereus and enables imaging with both structured illumination microscopy (SIM), and nano-scale secondary ion mass spectrometry (nanoSIMS). The platinum(II) moiety does not change the sub-cellular localisation or photophysical properties of the 4-amino-1,8-napththalimide dye. Given the readily tailored range of known napththalimide dyes, the platform reported here can be used to develop new probes targeted to specific bacterial imaging applications.

Acknowledgements: A.M.R. thanks Curtin University for the CIPRS Scholarship. M.V. and L.R. thank the Università degli Studi di Modena e Reggio Emilia for the More Overseas student exchange program that conferred the travel bursary to M.V.. We thank the University of Sydney for funding. We acknowledge the scientific and technical assistance of the Australian Microscopy and Microanalysis Research Facility at the Australian Centre for Microscopy and Microanalysis (ACMM). This work used computational resources of the CCIPL installed in Nantes. H.J. is supported by an Australian Research Council Discovery Early Career Researcher Award. The authors thank Philip A. Gale for useful discussions.

Conflicts of interest: There are no conflicts to declare.

\section{Notes and references}

1. H. W. Boucher, G. H. Talbot, J. S. Bradley, J. E. Edwards, D. Gilbert, L. B. Rice, M. Scheld, B. Spellberg and J. Bartlett, Clin. Infec. Dis., 2009, 48, 1-12.

2. (a) O. Kocaoglu and E. E. Carlson, Nat. Chem. Biol., 2016, 12, 472-478; (b) Z. Yao and R. Carballido-López, Ann. Rev. Microbiol., 2014, 68, 459-476.

3. (a) C. Coltharp and J. Xiao, Cellular Microbiol., 2012, 14, 1808-1818; (b) J. P. Schneider and M. Basler, Philos. Trans. R. Soc. B: Biol. Sci., 2016, 371, 20150499.

4. (a) L. Li, Y. Li, T. M. Lim and S. Q. Pan, FEMS Microbiol. Lett., 1999, 179, 141-146; (b) X. Ning, S. Lee, Z. Wang, D. Kim, B. Stubblefield, E. Gilbert and N. Murthy, Nature Mater., 2011, 10, 602-607; (c) L. C. Ong, L. Y. Ang, S. Alonso and Y. Zhang, Biomaterials, 2014, 35, 2987-2998; (d) A. M. Ranieri, C. Caporale, V. Fiorini, P. Rigby, S. Stagni, E. Watkin, M. I. Ogden, M. J. Hackett and M. Massi, Chem. Eur. J., 2019, 25, 10566-15070; (e) C. Reimold, H. J. Defeu Soufo, F. Dempwolff and P. L. Graumann, Molec. Biol. Cell, 2013, 24, 2340-2349; (f) P. Shieh, M. S. Siegrist, A. J. Cullen and C. R. Bertozzi, Proc. Nat. Acad. Sci., 2014, 111, 5456-5461; (g) P. Stiefel, S. Schmidt-Emrich, K. ManiuraWeber and Q. Ren, BMC Microbiol., 2015, 15, 1-9; (h) A. G. White, N. Fu, W. M. Leevy, J.-J. Lee, M. A. Blasco and B. D. Smith, Bioconjug. Chem., 2010, 21, 1297-1304; (i) C. Zhang, L. Yang, Y. Ding, Y. Wang, L. Lan, Q. Ma, X. Chi, P. Wei, Y. Zhao, A. Steinbchel, H. Zhang and P. Liu, Nature Commun., 2017, 8, 15979.

5. K. Ritchie, Y. Lill, C. Sood, H. Lee and S. Zhang, Philos. Trans. R. Soc. B: Biol. Sci., 2013, 368, 1-8.

6. (a) R. T. Schirra Jr. and P. Zhang, Curr. Protoc. Cytom., 2014, 70, 12.36.11-12.36.10; (b) E. Brown, J. Mantell, D. Carter, G. Tilly and P. Verkade, Semin. Cell Dev. Biol., 2009, 20, 910-919; (c) J. Caplan, M. Niethammer, R. M. Taylor and K. J. Czymmek, Curr. Opin. Struct. Biol., 2011, 21, 686-693; (d) K. Cortese, A. Diaspro and C. Tacchetti, J. Histochem. Cytochem., 2009, 57, 1103-1112; (e) S. Halary, S. Duperron and T. Boudier, Appl. Environ. Microbiol., 2011, 77, 4172-4179; (f) S. G. Li, G. Ji, Y. Shi, L. H. Klausen, T. X. Niu, S. L. Wang, X. J. Huang, W. Ding, X. Zhang, M. D. Dong, W. Xu and F. Sun, J. Struct. Biol., 2018, 201, 63-75; (g) J. M. Plitzko, A. Rigort and A. Leis, Curr. Opin. Biotechnol., 2009, 20, 83-89; (h) P. Schellenberger, R. Kaufmann, C. A. Siebert, C. Hagen, H. Wodrich and K. Grnewald, Ultramicroscopy, 2014, 143, 41-51; (i) E. Brown and P. Verkade, Protoplasma, 2010, 244, 91-97.

7. (a) P. Agüi-Gonzalez, F. Opazo, K.-A. Saal, S. Jähne, S. Kabatas, N. T. N. Phan and S. O. Rizzoli, Angew. Chem. Int. Ed., 2019, 1-7; (b) C. Höschen, F. Opazo, S. O. Rizzoli, U. Diederichsen, I. C. Vreja, K. Kröhnert, S. Kabatas and S. K. Saka, Angew. Chem. Int. Ed., 2015, 54, 5784-5788; (c) S. K. Saka, A. Vogts, K. Kröhnert, F. Hillion, S. O. Rizzoli and J. T. Wessels, Nat. Commun., 2014, 5, 1-8.

8. (a) A. C. Komor and J. K. Barton, Chem. Commun., 2013, 49, 3617-3630; (b) L. E. Wedlock, M. R. Kilburn, J. B. Cliff, L. Filgueira, M. Saunders and S. J. Berners-Price, Metallomics, 2011, 3, 917-925.

9. (a) B. K. Keppler, M. Galanski, A. A. Legin, I. Lichtscheidl, A. SchintImeister, M. Wagner and M. A. Jakupec, Chem. Sci., 2014, 5, 3135-3143; (b) M. T. Proetto, C. E. Callmann, J. 
Cliff, C. J. Szymanski, D. Hu, S. B. Howell, J. E. Evans, G. Orr and N. C. Gianneschi, ACS Central Sci., 2018, 4, 1477-1484.

10. (a) D. M. Doughty, M. Dieterle, A. L. Sessions, W. W. Fischer and D. K. Newman, PLoS ONE, 2014, 9, 1-8; (b) N. Musat, R. Foster, T. Vagner, B. Adam and M. M. M. Kuypers, FEMS Microbiol. Rev., 2012, 36, 486-511.

11. (a) E. E. Langdon-Jones, C. F. Williams, A. J. Hayes, D. Lloyd, S. J. Coles, P. N. Horton, L. M. Groves and S. J. A. Pope, Eur. J. Inorg. Chem., 2017, 2017, 5279-5287; (b) E. E. Langdon-Jones, N. O. Symonds, S. E. Yates, A. J. Hayes, D. Lloyd, R. Williams, S. J. Coles, P. N. Horton and S. J. A. Pope, Inorg. Chem., 2014, 53, 3788-3797.

12. (a) G. W. V. Cave, N. W. Alcock and J. P. Rourke, Organometallics, 1999, 18, 1801-1803; (b) G. W. V. Cave, F. P. Fanizzi, R. J. Deeth, W. Errington and J. P. Rourke, Organometallics, 2000, 19, 1355-1364; (c) A. M. Ranieri, L. K. Burt, S. Stagni, S. Zacchini, B. W. Skelton, M. I. Ogden, A. C. Bissember and M. Massi, Organometallics, 2019, 38, 1108-1117.

13. K. G. Leslie, D. Jacquemin, E. J. New and K. A. Jolliffe, Chem. Eur. J., 2018, 24, 5569-5573.

14. (a) J. R. Berenguer, E. Lalinde and J. Torroba, Inorg. Chem., 2007, 46, 9919-9930; (b) S. Fuertes, S. K. Brayshaw, P. R. Raithby, S. Schiffers and M. R. Warren, Organometallics, 2012, 31, 105-119; (c) M. Krause, D. Kourkoulos, D. Gonzlez-Abradelo, K. Meerholz, C. A. Strassert and A. Klein, Eur. J. Inorg. Chem., 2017, 5215-5223; (d) S. C. F. Kui, S. S. Y. Chui, C. M. Che and N. Zhu, J. Am. Chem. Soc., 2006, 128, 8297-8309; (e) S. C. F. Kui, F. F. Hung, S. L. Lai, M. Y. Yuen, C. C. Kwok, K. H. Low, S. S. Y. Chui and C. M. Che, Chem. Eur. J., 2012, 18, 96-109; (f) W. Lu, M. W. C. Chan, K.-K. Cheung and C.-M. Che, Organometallics, 2001, 2477-2486.

15. (a) S. Banerjee, J. A. Kitchen, S. A. Bright, J. E. O'Brien, D. C. Williams, J. M. Kelly and T. Gunnlaugsson, Chem. Commun., 2013, 49, 8522-8524; (b) R. M. Duke, E. B. Veale, F. M. Pfeffer, P. E. Kruger and T. Gunnlaugsson, Chem. Soc. Rev., 2010, 39, 3936-3953; (c) H. Guo, M. L. Muro-Small, S. Ji, J. Zhao and F. N. Castellano, Inorganic Chemistry, 2010, 49, 6802-6804; (d) F. Zhong and J. Zhao, Eur. J. Inorg. Chem., 2017, 2017, 5196-5204.

16. (a) D. Jacquemin, B. Moore, C. Adamo and J. Autschbach, J. Chem. Theory Comput., 2014, 10, 1677-1685; (b) D. Jacquemin, A. Planchat, C. Adamo and B. Mennucci, J. Chem. Theory Comput., 2012, 8, 2359-2372.

17. I. Maslov, A. Bogorodskiy, A. Mishin, I. Okhrimenko, I. Gushchin, S. Kalenov, N. A. Dencher, C. Fahlke, G. Bldt, V. Gordeliy, T. Gensch and V. Borshchevskiy, Sci. Rep., 2018, 8, 1-12. 